\title{
Approche théorique et expérimentale du fonctionnement d'un système de barrières pare-congères
}

\author{
Theoretical and experimental approach \\ to the functioning of a snow drift barrier system
}

G. Brugnot

CEMAGREF

B.P. 76, 38402 Saint-Martin-d'Hères

Le phénomène de mise en suspension, de transport et de dépôt d'un matériau granulaire par le vent a occupé un grand nombre de chercheurs, il suffit pour s'en convaincre de consulter certaines bibliographies, par exemple celle de Diounine qui dans un ouvrage cite 343 références (Diounine, 1963).

Les études les plus anciennes ont été effectuées sur le sable et les premiers chercheurs qui se sont intéressés aux phénomènes du transport de la neige sont partis des travaux de Bagnold (Bagnold, 1941/réédité en 1973).

Un domaine voisin est celui du transport solide en milieu aqueux. La partie de théorie commune est celle qui correspond aux conditions de " mise en suspension " d'un matériau granulaire.

Sur le plan théorique comme sur le plan expérimental, les travaux dans le domaine du transport de la neige par le vent ont d'abord concerné les régions polaires, puis les pays continentaux froids (Canada, URSS, USA).

Les pays alpins se sont assez peu intéressés à ces questions et pourtant non seulement ils ont des problèmes de congères, mais de plus le transport de la neige par le vent est une cause fréquente d'avalanche. Il est certain que l'étude du phénomène est beaucoup plus compliquée en présence du relief.

Nous nous proposons dans ce qui suit de faire une synthèse des connaissances dans le domaine du transport de la neige par le vent, puis de montrer comment nous avons cherché à adapter ces acquis à des conditions typiquement française grâce à une approche expérimentale.

\section{Les mécanismes du transport de la neige par le vent}

Dans les zones couvertes de neige, on observe fréquemment des phénomènes de transport qui se manifestent, par exemple, par des réductions de visibilité. Selon que le vent est plus ou moins violent, ce transport - et la réduction de transparence de l'air qui en résulte - s'effectue à des niveaux différents. On passe d'un transport au sol à un transport pouvant s'effectuer sur plusieurs dizaines de mètres.

L'analyse physique des phénomènes conduit à la mise en évidence de trois mécanismes fondamentaux :

- la reptation;

- la saltation;

- la diffusion turbulente.

Ces trois stades correspondent à des vitesses de vent croissantes, mais d'autres paramètres interviennent, par exemple ceux qui conditionnent l'arrachement du grain de neige en place : répartition des grains selon la forme et la dimension, état de transformation de la neige, etc. Nous considérerons le fait du transport comme une donnée, définie par un débit horizontal de neige.

La reptation intervient pour des vents faibles, elle consiste en un déplacement des grains de neige dans le sens du vent le long de la surface et ceci sans décollement appréciable. Elle s'explique assez bien par l'action directe du vent dans la sous couche laminaire.

La reptation nous concerne peu dans le cadre que nous nous sommes fixé. Elle intervient probablement dans les formes d'érosion observables à la surface de la neige.

La saltation apparaît à partir de vitesses de vent de l'ordre de $10 \mathrm{~m} / \mathrm{s}$ (mesurée à 10 mètres du sol). Elle est caractérisée par une trajectoire disymétrique des particules 
qui s'élèvent verticalement sur quelques centimètres, puis " retombent " selon une courbe parabolique. Ce phénomène a donné lieu à des explications très controversées, aucune ne paraissant très satisfaisantes. Une hypothèse souvent avancée est celle de l'impact des particules qui, à l'arrivée à la surface, communiqueraient une certaine quantité de mouvement à cette surface, d'où résulterait une mise en mouvement de grains en place. Cette hypothèse paraît réaliste pour le sable (encore que se pose le problème de l'initiation du phénomène), mais moins pour la neige qui paraît mal se prêter aux chocs élastiques (Mellor, 1965).

D'autres auteurs mettent en avant une pression d'arrêt liée à des tourbillons situés près du sol (Diounine, 1963). Cette hypothèse nous paraît plus crédible. En effet, les observateurs s'accordent pour reconnaître que la saltation concerne une tranche d'écoulement comprise entre quelques centimètres et un mètre du sol : compte tenu de la très faible rugosité du manteau neigeux, on est nettement dans une zone d'écoulement turbulent. Une autre explication partielle peut être cherchée, à notre avis, dans le phénomène de la portance, qui peut suffire à expliquer le décollement de grains s'ils ont une forme plate et sont placés de certaines façons. Quoiqu'il en soit, il est probable que la saltation est le principal mode de transport de neige par le vent hors des régions polaires.

La diffusion turbulente est, comme son nom l'indique, un phénomène de diffusion lié à la turbulence qui, nous l'avons vu précédemment, joue un rôle très important dans le domaine du transport de la neige par le vent. Elle correspond à un transport à des altitudes plus élevées que dans le cas de la saltation et surtout les interactions avec la surface sont faibles. Si l'on s'en tient à cette définition, la diffusion turbulente ne joue aucun rôle dans la formation des congères. En revanche, de même que ce mode de transport de la neige est généralement constitué comme jouant un rôle important dans le domaine du transport de neige à distance dans l'Antarctique, il intervient certainement dans la répartition à grande échelle du manteau neigeux dans les montagnes de l'Europe tempérée.

\section{Les ouvrages à vent}

\section{Mode d'action de ces ouvrages}

Le transport par saltation s'effectue de façon uniforme sur une surface plane et dépourvue d'obstacles. Il y a donc un équilibre entre les quantités de neige déposées, d'où une absence d'accumulation. Mais, dès qu'un obstacle intervient, il y a séparation de la couche limite turbulente et formation d'un sillage sous le vent de l'obstacle. Ce sillage présente deux caractéristiques importantes. D'une part, la vitesse de frottement diminue (elle peut s'inverser localement), donc la capacité d'érosion de l'écoulement s'annule pratiquement. D'autre part, il constitue un véritable piège à neige, car quand les particules entrent dans cette zone, elles ne peuvent plus progresser par saltation puisqu'il n'y a plus de composante du vent uniforme et parallèle à la pente, mais une série de tourbillons si bien que la particule finit par se déposer : il se forme une congère.
Nous n'insisterons pas sur les inconvénients liés à la formation de ces congères, ceux-ci sont bien connus dans le domaine de la circulation. Nous allons plutôt examiner les parades qui permettent d'atténuer ce type de nuisance. Le principe de ces parades se déduit de la description que nous venons de faire du phénomène.

On doit d'abord considérer si l'on peut éliminer la cause première, à savoir la singularité provoquant la séparation de l'écoulement. C'est parfois possible, il suffira de supprimer un muret de pierres ou d'araser une crête de talus. C'est souvent impossible, un cas fréquent est celui de routes que l'on a du tracer en déblai/remblai et qui présentent certains tronçons en déblai perpendiculaires au vent qui mobilise la neige. Dans ce cas, il faut provoquer une séparation d'écoulement à l'amont de la zone "critique ". C'est le principe d'action de la plupart des ouvrages à vent.

Il existe un troisième type de méthode qui consiste à modifier l'effet de la singularité, non pas en la faisant disparaître physiquement, mais en accélérant artificiellement l'air à son voisinage. Ces ouvrages, dits "souffleurs ", s'appliquent mal dans le domaine routier, pour des raisons essentiellement pratiques. Ils ont été utilisés en haute montagne avec des fortunes très diverses.

\section{Description des ouvrages utilisés}

Nous présenterons les barrières à neige. Celles-ci sont très utilisées en haute montagne pour la lutte contre les avalanches. En outre, elles représentent la quasi-totalité des ouvrages rencontrés dans le cas de la protection des routes, qui est précisément le sujet sur lequel est centré cet article.

Une barrière à neige provoque une séparation artificielle de l'écoulement et son principal intérêt est que l'on peut faire facilement varier son effet en agissant sur un certain nombre de paramètres, parmi lesquels la garde au sol (espace laissé vide au pied de l'ouvrage), la porosité (pourcentage de vides vu par l'écoulement) et d'une façon plus générale, la forme des vides, la hauteur, l'inclinaison, etc. Sous le vent d'une barrière, on a donc une accumulation "dirigée ", la neige ainsi interceptée est assez difficilement mobilisable du fait de la cohésion qu'elle acquiert lors de son dépôt, si bien que même si la direction du vent change, l'expérience montre que la neige accumulée par une barrière à vent est difficilement reprise.

Nous allons illustrer le fonctionnement de barrières à neige à partir d'un cas concret, celui du périmètre expérimental de Besse-en-Chandesse.

Le périmètre d'essai de Besse-en-Chandesse

\section{Objectifs poursuivis}

Le Ministère des Transports a financé en 1982 la mise en place d'un important dispositif d'essai de barrières à neige dans le département du Puy-de-Dôme. La participation des services régionaux et locaux de l'Equipement (C.E.T.E., Subdivision) et du C.E.M.A.G.R.E.F., a permis de définir et de réaliser un protocole de mesure nivo- 
météorologiques. Les résultats ont fait l'objet d'une publication intégrale (CETE/CEMAGREF, 1983). Nous ne reprendrons que quelques éléments indispensables à l'interprétation.

Le terrain utilisé se trouve à une altitude d'environ $1300 \mathrm{~m}$; on n’a pas pu éviter de légères irrégularités de relief mais dans l'ensemble, les pentes locales qui ne dépassent pas $1,5 \%$, sont exposées " au vent ".

7 types de barrières ont été testées, toutes alignées selon une longueur totale de 175 mètres. Les caractéristiques de ces barrières ont été choisies de façon à pouvoir faire un certain nombre de comparaisons et mettre en évidence l'effet des facteurs suivants: porosité, garde au sol, hauteur, type de matériau employé, orientation. Compte tenu des objectifs très pratiques de l'opération, on a certes suivi le fonctionnement des ouvrages, mais on a également enregistré toutes les données concernant le coût des barrières, les problèmes pratiques posés par leur mise en place et leur entretien. L'objectif était de définir quel type de barrière apportait une réponse à un problème donné et ceci au moindre coût.

\section{Résultats de la campagne 1982-1983}

Nous ne présenterons pas de façon systématique les performances des différentes barrières à neige, ce qui fait l'objet du rapport d'essai déjà cité, mais nous essayerons d'établir un lien entre les résultats observés et les éléments de théorie exposés au début du présent article, ceci dans le but de mieux comprendre ces résultats et d'améliorer le fonctionnement des barrières à neige traditionnelles.

Point $n^{\circ} 1$ : Effet de la garde au sol: un espace doit être laissé entre le sol et le pied de la barrière et ceci pour éviter d'intercepter de façon trop brutale la couche de saltation. Il semble en effet souhaitable de ne pas trop modifier les conditions à l'amont de l'ouvrage, c'est-à-dire de ne pas laisser trop de neige s'accumuler car l'efficacité de la barrière se réduit. Par ailleurs, il faut éviter que la base d'une barrière à vent soit prise dans la neige, car le tassement de celle-ci endommagera l'ouvrage. Le problème est plus critique pour les barrières à lattes horizontales, qui obstruent des tranches d'écoulement d'épaisseur donnée, tandis que les barrières à lattes verticales laissent passer des " profils " d'écoulements entiers.

Cet effet se vérifie sur les barrières testées, car celles dont les bases sont les plus obstruées sont d'abord une barrière sans garde au sol, puis une barrière où pour des raisons pratiques la garde au sol réelle est variable et devient presque nulle au milieu d'une portée.
Point no 2: Effet de la porosité: plus la porosité d'une barrière croit, plus elle diffuse la neige de façon efficace. Ceci se traduit par un allongement de la congère, une diminution de sa hauteur, un éloignement du maximum, mais une augmentation du volume stocké. En outre, le volume de neige intercepté à l'amont se réduit, et là l'effet de porosité se superpose à l'effet de garde au sol. Cet effet est particulièrement net dans les résultats obtenus, puisque la seule barrière ayant une porosité supérieure à $50 \%$ accumule une congère présentant toutes les caractéristiques énumérées ci-dessus: congère plus longue, moins épaisse, avec un maximum situé si loin que la congère est presque symétrique. Par ailleurs, c'est celle qui conduit au volume adimensionnalisé (en divisant par le carré de la hauteur de la barrière) le plus élevé. Enfin, c'est celle qui forme la congère la plus réduite du côté au vent.

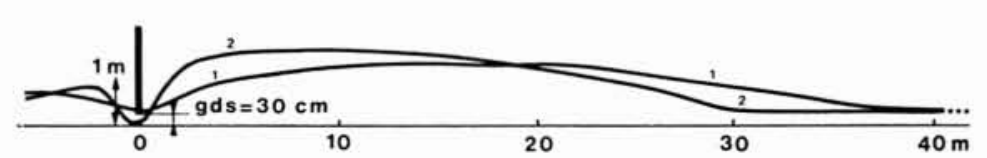

Figure 2 - Effet de la porosité

1. porosité $\simeq 70 \%$

2. porosité $=50 \%$

Point no 3 : Effet de la hauteur : il peut paraître surprenant que nous n'ayons pas commencé par ce paramètre et pourtant il semble que celui-ci ne joue pas un rôle très net. Une barrière de hauteur totale $1,50 \mathrm{~m}$ a mal fonctionné, c'est-à-dire qu'elle a été enfouie partiellement sous la neige malgré la présence d'une garde au sol de $30 \mathrm{~cm}$. Toutes les autres, mesurant $1,80 \mathrm{~m}$ ou plus, n'ont pas subi ce sort, même quand leur base a été un peu prise du fait de l'absence de garde au sol. On peut expliquer ce phénomène de hauteur critique en remarquant qu'une barrière à neige ayant une géométrie donnée a une capacité de stockage donnée. Des essais sur modèle réduit ont montré que cette capacité correspondait à peu près au volume du sillage engendré par l'ouvrage, lequel varie à peu près comme le carré de la hauteur (c'est un volume par mètre linéaire de barrière, donc de dimension $L^{2}$ ). Quand cette capacité est atteinte, la barrière à neige évolue assez rapidement vers une obstruction par l'amont. Au-delà de la valeur critique correspondant à la hauteur de barrière exactement nécessaire pour stocker dans de

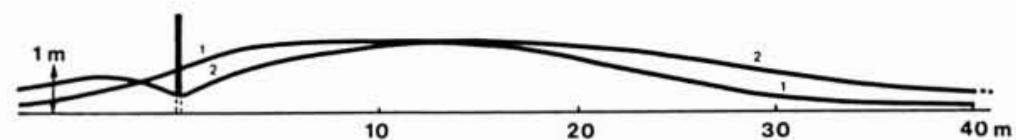

10
Figure 1 - Effet de la garde au sol

1. garde au sol $=0$

2. garde au sol $=30 \mathrm{~cm}$

(porosité : $75 \%$ ) 
bonnes conditions l'apport de la neige de l'hiver, une variation de hauteur ne se traduit pas par une amélioration d'efficacité : c'est ce que nous avons constaté; alors le paramètre hauteur est moins important que le paramètre porosité.

Point no 4 : Comparaison des barrières testées: les renseignements recueillis nous ont permis de classer les barrières selon leurs performances exprimées d'une part en termes purement physiques, d'autre part du point de vue efficacité-coût. Les barrières les plus intéressantes sur le plan physique sont les barrières en chataignier refendu, du fait de leur porosité élevée et de leurs lattes verticales. Ce sont aussi les meilleures dans l'optique coût-efficacité, mais les barrières en matière synthétique ont un avenir certain dans les cas où une dépose annuelle est nécessaire, car ce sont celles qui se prêtent le plus facilement à cette opération coûteuse en terme de main-d'œuvre. Il est donc important d'améliorer leur efficacité dans l'accumulation de la neige.
Point $n^{\circ} 5$ : Discussion des formules de calcul de barrières à neige : une saison de mesure ne permet pas d'établir des formules de type statistique. Il est cependant très intéressant de comparer les résultats obtenus avec ceux qui sont admis et qui ont été obtenus soit par modélisation, soit par mesure de terrain. On retrouve sur deux points un accord excellent avec les résultats soviétiques (Diounine, 1963) et américains (Tabler, 1974) : à la saturation, $\mathrm{L} / \mathrm{H}$ (longueur adimensionnalisée de la congère) est de l'ordre de 25 et $\mathrm{A} / \mathrm{H} 2$ (surface adimensionnalisée) est de l'ordre de 20. Ces deux formules nous ont toujours paru conformes à l'observation, mais comme elles contredisaient plusieurs formules admises en Europe, un certain doute subsistait, que les mesures faites à Besse en Chandesse nous ont permis de lever. Notre explication de ces formules erronées est qu'elles reposent sur des observations faites loin de la saturation. En effet, elles fournissent toutes des valeurs insuffisantes pour la longueur et le volume de la congère.

\section{Conclusion}

La première campagne de mesures effectuée sur le site expérimental de Besse-en-Chandesse a permis de vérifier l'efficacité de la barrière à neige dans la prévention des congères. Certains ont pu émettre des doutes sur ce point, mais il nous a toujours semblé que le mauvais fonctionnement des barrières à neige que nous avons observées était lié à une mauvaise implantation et à un mauvais choix des caractéristiques des ouvrages posés. Les mesures faites à Besse, qui ont été poursuivies pendant l'hiver $1983 / 1984$, donnant des indications extrèmement précieuses qui devraient permettre d'éviter de grossières erreurs de conception.

\section{Références bibliographiques}

BAGNOLD R.A., 1973. - The physics of blown and desert dunes. Chapman \& Hall, London.

CETE/CEMAGREF., 1983. - Essais comparatifs de barrières à neige, hiver 1982/1983. Rapport interne.

DIOUNINE A.K., 1963. - Dynamique des blizzards (en russe). Publication de la section de Sibérie de l'Académie des Sciences d'U.R.S.S., Novossibirk.

MELLOR M., 1965. - Blowing snow. Cold régions science and engineering, Part. III, Section A3C. Publication du C.R.R.E.L. Hanover, New Hampshire.

TABLER R.D., 1974. - New engineering criteria for snow fence systems. Transp. Res. Rec., $\mathrm{N}^{\circ} 506$, p. 65-78. 


\section{Snow fences \\ Theoretical and experimental approaches \\ Gérard Brugnot, C.E.M.A.G.R.E.F.}

\section{Introduction}

As a granular material, snow is eroded, moved dowstream and eventually deposited under wind action. This phenomenon has been investigated for a long time, but we shall stress the important advances performed by Bagnold in the field of sand, which were useful for snow researchers. Shall we consider the question of any granular material in any fluid, we have to quote the problem of sediment transport in water.

Alpine countries are not as broadly concerned by drifting snow problems as the large subpolar regions of USSR and Canada, but in the former the wind action on snow is more and more considered as an important avalanche factor.

\section{Blown snow transport}

In snow-covered regions, there is a steady snow transport under wind action. This transport often reduces visibility and results in snowdrifts, i.e. snow accumulations in some locations. From observations and theoretical considerations, researchers distinguished three modes of blown snow transport : creeping, saltation and turbulent diffusion. These different modes seem to be in correspondance with the respective physical phenomenons : displacement in laminar sub-layer, air-lift and gravity effects with strong interactions between grains and snow surface, turbulent transport of microscopic grains.

\section{Snowdrifts formation and prevention}

As far as a flat and protrusion-free surface is concerned, saltation develops uniformly, without any local accumulation. But if there is any cause of flow separation, we observe how a wake is present with some large eddies within. In this region, snow particules no more bounce forward, because the average air velocity is of the same order as their fall velocity : they settle. Unfortunately, the resulting snow accumulation causes undesirable obstructions in some cases, e.g. around building, on roads...

The best preventive action is to build "something " which, beeing properly designed and located, will cause an artificial separation of the air flow and such way a snow settlement in a region of non particular interest. Formerly, people used to build some "walls " to " stop " the drifting snow, but this solution presents several drawbacks, i.e. they accumulate a rather small amount of snow and they rapidly disappear inside the snowdrifts, which results in large vertical pressure due to snow settlement : these walls were unefficient and expensive.
A drastic improvement consisted in replacing the walls by "porous " fences, which appeared as a progress if we consider the two above mentionned points : since they allow snow to flow across them and to deposit only leeward, they store a larger quantity of snow and the snowdrifts build at some distance of the fence, especially if the latter presents a bottom gap.

\section{The Besse en Chandesse experimental snow-fences field}

To be efficient, a snow fence is to be designed according to several criteria, some of them were already quoted : porosity, bottom gap. Other important features are total height, i.e. the scale factor, and of course the fence location. The influence of these factors is well known, but the French Ministère des Transports decided two years ago to fund an experimental field, first to verify generally admitted rules in typical Central France conditions, then to test several fences available in France from a cost/ efficiency point of view.

An interesting location was found in the Puy de Dome department, where 7 different snow fences were built, the total system length being 175 meters. The experiment is planned along several years but the first winter provided some interesting resuls :

* classical Chestnut fences got the best cost/efficiency results

* synthetic fences are easily placed and removed but too expensive and not so efficient

* experiments of "adjustable bottom gap " made on horizontal slat fences were encouraging

* some "self-orienting" snow-fence had interesting results

* the generally admitted Soviet, U.S. and Canadian formulas are verified provided a sufficient amount of drifting snow is available and the fences are not too high : when saturation is reached, adimensionalized snowdrift distance is around 25 and adimensionalized area around 20 . Other formulas, especially Swiss and Austrian ones, which provide significantly lower values do not account for saturation condition.

\section{Conclusion}

We intend to go on with the Besse en Chandesse observations, trying different fence shapes and sizes, having in mind a cost/efficiency preoccupation. As for technological breakthroughs, we consider that after the design of a self-orienting snow-fence, there is an obvious need of progress in the bottom-gap problem. 


\section{Discussion}

Président : M. L. de CRÉCY

M. le Président remercie Monsieur Brugnot et ouvre la discussion :

M. Lliboutry. - Je m'étonne qu'on ne parle pas de consolidation de la neige et qu'on assimile encore la neige à du sable.

M. BRUGNOT. - Le problème est très complexe. Labsence de mesures sérieuses sur la neige entretient les controverses entre chercheurs.

M. Lliboutry. - La consolidation de la neige et son durcissement, rien de tout cela n'a d'équivalent dans le sable. Il se forme une pellicule de glace transparente d'un millimètre ou plus d'épaisseur et qui donne son aspect particulier aux sommets ventés. C'est cela qui permet ensuite la corrosion et la formation des sastrugis.

M. BRUGNOT. - Dans le cas de la congère ventée, c'est la théorie; dans la pratique, les phénomènes sont complexes.

M. ROBERT rappelle l'installation d'une barrière pare-neige en 1938 dans les Pyrénées Orientales au Col de La Quillane. Cet ouvrage fonctionnait parfaitement. L'effet du pare-neige était déterminant dans la décharge de la neige. Le vent provoquait en effet le dépôt de celle-ci à un emplacement bien déterminé à l'aval du pare-neige. La garde au sol n'a pas soulevé de difficultés.

Peut-être avons-nous eu de la chance?

M. le Président. - La constance de la direction du vent jouait favorablement dans ce cas. 